\title{
The Role of Unlicensed in Spectrum Reform
}

\author{
William Lehr \\ Massachusetts Institute of Technology \\ (wlehr@mit.edu) \\ ** March 17, 2005 **
}

\section{Introduction}

In many countries, there is a growing consensus among industry participants, academics, and policymakers that traditional models for managing radio frequency (RF) spectrum are badly outdated. ${ }^{1}$ The existing management regime is premised on century old radio technology. Under the traditional approach, regulators allocate narrow frequency bands to specific uses and users under restrictive licenses that constrain the choice of technology, business model, and ability to redeploy the spectrum to higher value uses or to make use of new technologies. This approach has resulted in acute spectrum scarcity. This scarcity is largely artificial in that it results from an outmoded regulatory regime, rather than because of any technical or market capacity constraints.

This artificial scarcity distorts the opportunity cost of spectrum. New technologies and uses face excessively high costs for accessing spectrum. It is is either not available at all or only after the payment of high auction fees. Meanwhile, incumbents (e.g., government users and over-the-air broadcasters) face excessively low opportunity costs for the spectrum they control, which provides them little incentive to invest in enhancing spectral efficiency. Appropriate regulatory reform can eliminate this artificial scarcity which ought to result in more efficient spectrum use and lower prices for spectrum overall. $^{2}$

Economists are generally agreed that substituting market forces for direct government management of spectrum use (the so-called legacy "command and control" model) will enhance efficiency and promote innovation. ${ }^{3}$ There are two basic regulatory models for reforming spectrum management to be more responsive to market forces: the "flexible licensed" and the "unlicensed" models. ${ }^{4}$ Most economists who have focused on

\footnotetext{
${ }^{1}$ See Kolodzy (2002) or Ofcom (2004).

${ }^{2}$ Eliminating artificial constraints on spectrum which currently block entry of new wireless technologies and services (and constrain the expansion of existing ones like mobile services) will expand total demand also so it is not possible to predict a priori what the future equilibrium marginal price for spectrum will be. However, there are many technical innovations that have yet to be widely adopted that could greatly expand the capacity of wireless systems (e.g., smart radios systems, new modulation and signal processing techniques). Reforms that encourage the adoption of such technologies will also expand the "marginal supply" of accessible spectrum.

${ }^{3}$ See Comments of 37 Concerned Economists (2001).

${ }^{4}$ I refer to "flexible licensing" to distinguish the new model from the legacy model which also uses spectrum licenses to manage access.
} 
the issue favor the "flexible licensed" model for the spectrum that is currently perceived to be the most valuable, and hence most "scarce." This is the lower frequency spectrum below $3 \mathrm{GHz}$ (and even more valuable, below $1 \mathrm{GHz}$ ). ${ }^{5}$ Policymakers at the FCC and Ofcom have concurred in this view and current plans emphasize a flexible licensed approach for this lower frequency spectrum. ${ }^{6}$

This essay makes the counter case for why the unlicensed model is also important and should be relied on to manage a greater share of the high-value lower frequency spectrum. Because it is neither desirable nor politically feasible $e^{7}$ to advocate adopting the unlicensed model instead of the flexible licensed model, this paper makes the case for unlicensed spectrum in a world where it is assumed that the majority of high-value spectrum is managed via flexible licensing. It explains why an over-reliance on the "flexible licensed" model - reflected in a failure to provide for additional "unlicensed" use in lower frequency spectrum -- will hamper progress toward eliminating artificial spectrum scarcity. This, in turn, will hamper the evolution of wireless technology and markets, and limit progress toward effective regulatory reform.

The balance of the essay is organized into three sections. In Secion II, I explain key differences and commonalities in the licensed and unlicensed approaches. Section III then focuses on why the unlicensed model offers important benefits for regulatory reform. Section IV concludes and discusses challenges with expanding the unlicensed use further.

\section{Flexible Licensed and Unlicensed Spectrum Management Explained}

While there is a growing consensus that transitioning from the legacy regulatory model, caricaturized as "command and control," toward market-based spectrum management is desirable, there is no general agreement as to how this should be accomplished. ${ }^{8}$ The two principal approaches under consideration are the "flexible

\footnotetext{
${ }^{5}$ For leading economists supporting the "flexible licensed" model, see Cave (2002), Faulhaber and Farber (2002), Hazlett (2001), or Kwerel and Williams (2002). Among the economists, exceptions include Lehr (2004) and Noam (1995). Support for the unlicensed model has been lead by engineers and lawyers (see, e.g., Benkler (2002), Lessig (2001), Reed (2002), and Werbach (2003)).

${ }^{6}$ See Kolodzy (2002) or Ofcom (2004).

${ }^{7}$ There are still many issues (technical, business, and policy) associated with adopting an unlicensed management approach that need to be worked out and movement to unlicensed as the sole approach would represent far too dramatic a departure from the status quo for it to even be politically feasible.

${ }^{8}$ I refer to the labeling of spectrum management models as "caricatures" because the simple taxonomy overstates the complexity in rules, and understates the significant progress that the FCC and other regulatory bodies have made in reforming rules for at least certain bands over time. For example, the PCS mobile telephone bands in the United States were first licenses to be auctioned. They allow licensees flexible choices with respect to the technology adopted, the services offered, and other features regarding how the spectrum is used.
} 
licensed" and "unlicensed" models. The "flexible licensed" model is sometimes referred to as the "market mechanism," "exclusive use," or "property rights" approach; while the latter, is sometimes referred to as the "license-exempt," "open," "free," or "commons" approach.

\section{A. A false dichotomy}

These short-hand labels are intended to emphasize alleged distinguishing features, but as with most such labels, they conflate multiple issues that suggest an overly simplistic and misleading dichotomy. On the one hand, the licensed/property rights model is portrayed as more consistent with a market approach that treats spectrum as a scarce resource that is amenable to being traded in secondary markets once property rights are assigned. This is intended to contrast with the unlicensed/open/commons model which views spectrum as belonging to everyone, and hence, not amenable to such an assignment of property rights or trading. According to this stylized perspective, the "licensed" model recognizes that spectrum is "scarce," and that its allocation would be more efficient if it were first "auctioned" and then traded in "markets." In contrast, the "unlicensed" approach views whatever spectrum scarcity exists as wholly artificial, and therefore, access should be free to all, with the government establishing the framework to manage such access.

With this simplistic caricature of the debate, it is not surprising that most economists and current mainstream regulatory policy - which favors transitioning to markets wherever possible - favor the licensed approach for the most valuable spectrum. This is unfortunate because the dichotomy described above is more apparent than real.

First, both models represent a transition to increased reliance on market forces, but neither model eliminates regulation of the airwaves. In the case of a licensing regime, it is anticipated that policymakers will assign flexible and tradable spectrum use rights to licensees, eliminating the many constraints that characterize and encumber the current licensing regime. However, how to define such rights optimally is far from clear. Additionally, policymakers need to foster the emergence of secondary markets if the transition to a flexible licensing regime is to deliver the promised efficiency enhancements. Regulatory reform takes time and while it may not be possible to precisely foresee what the eventual outcome will be, we can be sure that the actual outcome will deviate from the theoretical ideal. Finally, there is the expectation that courts will substitute for administrative agencies in enforcing license property rights, but there is no a priori reason to believe that this will result in lower costs (greater immunity from influence costs). ${ }^{9}$

The view that unlicensed might be less deregulatory is ironic since an open access/unlicensed regime is much more deregulatory, at least in principle. Although it is

\footnotetext{
${ }^{9}$ Indeed, if spectrum courts are specialized administrative courts (which seems plausible), then they may be only marginally different from expert agencies with regards to their vulnerability to influence costs.
} 
unlikely that unlicensed spectrum would be managed without any regulation to limit interference, the protocol or rules could be chosen by users or industry (e.g. via industry standardization) and so need not require on-going government management of the technology choices for how spectrum is accessed. Both the flexible licensing and unlicensed regimes will require on-going regulation while at the same time offering greater scope for market forces.

Second, while the licensed approach only makes sense if one believes that spectrum will continue to be scarce - that is, have a non-zero opportunity cost -- support for the unlicensed approach does not require belief that all scarcity will be eliminated. Indeed, the choice of regulatory regime cannot eliminate real spectrum scarcity which may arise whenever there are more contending potential uses than mutually can be accommodated. When congestion arises in unlicensed spectrum, there needs to be a mechanism for dealing with it - but it is not true that licenses offer the unique best mechanism for allocating scarce spectrum in all situations. Congestion imposes an externality (e.g. in the form of lower quality performance) that has a real opportunity cost that influences behavior in ways similar to (if not identical to) the ways in which a market-clearing price for licensed spectrum would be expected to operate. There are lots of cases where society chooses alternative (non-price-based) mechanisms to allocate scarce resources (e.g., ambulances, public goods) and the "Tragedy of the Commons" often does not arise with collective resource management.

Third, auctions may (or may not) play a role in the transition to either licensed or unlicensed spectrum. Certainly, the hope for both models is that the transition will help reduce artificial spectrum scarcity and so dramatically reduce spectrum opportunity costs. Thus, if reform is successful, the expected proceeds from any auction should be substantially lower. This makes it both less necessary to allocate spectrum via auction and less costly to acquire unlicensed spectrum via auction. ${ }^{10}$ Indeed, while auctions may be important as part of the transition mechanism to market-based management, allocating additional spectrum for unlicensed could be incorporated in this framework (e.g., as a transition mechanism). ${ }^{11}$ Indeed, with the realization of effective reform, auctions will be less necessary to ensure efficient assignment (i.e., secondary market trading of licensed spectrum can achieve this) and to ensure fair sharing of the benefits from spectrum access (e.g., unlicensed and licensed co-existing and lower opportunity costs overall mean allocation of windfalls is less of an issue).

In summary therefore, both the flexible licensed and unlicensed regimes are deregulatory - without eliminating regulation; are consistent with scarcity - while anticipating that artificial scarcity will be greatly diminished; and are consistent with markets managing flexible use of the spectrum.

\footnotetext{
${ }^{10}$ As long as there are active secondary markets for spectrum and prices on those markets are not excessive, spectrum can be efficiently assigned regardless of whether it was originally auctioned or not.

${ }^{11}$ As in the "Big Bang" auction suggested by Kwerel and Williams (2002). Ikeda and Ye (2003) suggest a mechanism of spectrum buyouts to transition to open spectrum.
} 


\section{B. The real difference between licensed and unlicensed use}

The essential difference between the two regimes is that flexible licensing assigns an "exclusive use" property right to the licensee to transmit; whereas the unlicensed regime assigns a non-exclusive right to all potential users to transmit. The principle motivation for the exclusive use right is to protect the licensee's use of the spectrum from other, potentially interfering transmitters. By making this property right/license tradable and by eliminating constraints on how the licensee uses the spectrum (what services are offered or what technology is used), a flexible licensing regime induces the licensee to internalize the opportunity cost of spectrum scarcity.

In contrast, in the unlicensed/open use model, no potential user has an exclusive right to transmit. In a theoretically perfect world without transaction or adjustment costs, the unlicensed model may appear to offer less powerful incentives to use spectrum efficiently. However, in the imperfect world we actually inhabit with incomplete markets and where there are transaction and switching costs, concerns about market power and on-going regulation remains a reality, it is unlikely that social opportunity costs of spectrum use will be fully internalized by a flexible, exclusive-use licensee. An economic comparison of the licensed and unlicensed models is much more complex than suggested above.

First, the flexible licensing approach represents an incremental improvement over the existing regime which already uses licenses to manage the majority of commercial spectrum. In contrast, the unlicensed model represents a more radical form of reform. In light of the inherent bias in political processes in favor of the status quo, this induces an inherent bias in favor of flexible licensing over the unlicensed model. The former is closer to what we have always done and what industry and policymakers understand.

Second, under the flexible licensing regime, the licensee can exclude other users even if there is no real spectrum scarcity. ${ }^{12}$ That is, the licensee can choose to use its control of the license to induce artificial scarcity whenever that is consistent with its private interests (even when social welfare would be enhanced by allowing excluded users access). Certainly, in the past, a key feature of spectrum policy has been its use in shaping industry structure by limiting access to specific licensees. Hazlett (2001) documents the role of over-the-air broadcasters in the US in opposing the allocation of additional broadcasting licenses, and thereby slowing the adoption of technologies like UHF television and FM radio. Similarly, mobile carriers in some markets have opposed the allocation of additional 3G licenses, fearing the implications of excess competition. While it is certainly the hope that major reform, including the promotion of secondary

\footnotetext{
${ }^{12}$ Real spectrum scarcity arises when it would not be socially efficient to allow additional transmitters to share the spectrum. This may occur either for technical reasons (i.e., given the current state of technology, allowing the excluded transmitters would result in destructive interference) or for economic reasons (i.e., while it is technically feasible to allow the transmitters to share the spectrum, the total benefit would exceed the total cost of making the necessary technical adjustments).
} 
markets, will preclude the market power that makes such abuses both feasible and likely, this hope may be only imperfectly realized. If market power remains an issue, than the risk will remain that exclusive licenses will be used to enforce on-going artificial scarcity.

Third, the two models do imply different industry economics. The flexible licensing approach favors the network-centric service provider model; while the unlicensed approach favors the equipment-centric end-user model. The former implies more centralized management of spectrum while the latter is more distributed and decentralized. Which is better may depend on the circumstances and ones perspective. It is certainly no accident that most communication and broadcasting services are supported via businesses with a service-provider model (i.e., an integrated network that is owned and managed by single business entity is used to deliver services). This structure facilitates making coordinated decisions about management of the network and the spectrum that is used. This may facilitate the internalization of externalities over a wider region (e.g., when a provider offers wireless services over a metro-sized area) and if the network and frequency used are co-specialized (e.g., when the technology used is not frequency agile). New smart radio systems, however, make it increasingly feasible to decouple frequencies and radio networks and to manage interference in a decentralized fashion. With unlicensed spectrum, it is possible to build up a network from end-user equipment that can be linked in an ad hoc, wireless mesh. This supports viral, edge-based growth and offers an interesting alternative to the service-provider-based model that has traditionally dominated telecommunication services, including wireless services.

\section{Models for unlicensed access: primary vs. secondary use underlays and overlays}

Certainly, the flexible licensing regime represents an improvement over the current regime of inflexible licensing. As an incremental improvement, for a regulatory economist, it seems hard to oppose. ${ }^{13}$ Likewise, the complete abandonment of licensing in favor of unlicensed access for all spectrum seems far too radical a departure from existing practice to be either desirable or politically feasible. However, this latter approach is not even under consideration. Rather, mainstream spectrum reformers are advocating a mixed approach that makes use of both the licensed and unlicensed models. $^{14}$

While explicitly recommending a role for unlicensed, the mainstream reformers have relegated unlicensed to a secondary and more marginal role for the highest value, lower frequency spectrum. There are a number of good reasons for this outcome, which are worth considering, but they do not tell the whole story (as I explain in the next

\footnotetext{
${ }^{13}$ See Comments by 37 Concerned Economists (2001).

${ }^{14}$ Most countries still rely heavily on the legacy command and control model for managing spectrum, and if considering reform, are following a much more incremental approach. Among the countries that have adopted major reform agendas, the US (see Kolodzy, 2002) and UK (see Ofcom, 2004), are representative. The more radical approach of a complete transition to flexible licensing/property rights is relatively rare, with Guatemala an oft-cited example (see Hazlett, 2002); and no country as transitioned to a complete "unlicensed" model.
} 
section). There are two basic approaches for implementing unlicensed access: primary or dedicated access; and secondary access. There are two types of secondary access: underlays and overlays.

Underlay access allows secondary use of the spectrum by transmitters that operate at low power levels, in the noise floor of licensed spectrum. An example of such an underlay technology is Ultrawideband (UWB) that spreads the signal over a very wide bandwidth to limit the power emitted in any particular frequency band. The requirement to operate at low power limits the range of underlay devices. This fits well with the view that a "commons" management scheme is well-suited to managing spectrum use conflicts/interference among a few users/transmitters in a locally-concentrated area (e.g. within a home). There is a compelling need today for high-bandwidth wireless services to substitute for cabling (e.g., among stereo components) or to support WLANs. While it is certainly true that unlicensed has an important role to play in such situations, the converse is not universally true. While some wide area applications or providers may prefer the protection against interference afforded by an exclusive license, this is not necessary to ensure coordination and adequate interference protection.

The second type of secondary use "easement" is overlay access. An overlay device is allowed to transmit when the primary-use licensee is not using the spectrum. This could be defined in the spatial domain (e.g., rural areas that a broadcaster is not currently serving) or time domain (e.g., when the primary licensee is not transmitting using "listen-before-talking" technology). The unlicensed overlay devices would use appropriate sensing technology to determine when the spectrum is free to transmit. Collectively, the devices that are able to modify their operation dynamically in response to local RF conditions are referred to as "cognitive radios."15

As noted earlier, most mainstream policymakers advocate allocating most of the high-value lower-frequency spectrum to exclusive-use licensed primary users, with only minimal (if any) expansion of primary (dedicated) spectrum for unlicensed use. An important rationale for this approach is that unlicensed needs can be adequately met by secondary access via either overlays or underlays. ${ }^{16}$ There is an obvious appeal to this approach since all transmitters in primary (dedicated) unlicensed spectrum are secondary users since no one has a right to exclude any other qualified user. In contrast, with licensed spectrum, the licensee with the exclusive right clearly has a primary claim to the spectrum.

While appealing, there are several problems with this view. First, the attempt to define overlays or underlays represent attempts to circumscribe the licenses to limit their scope so that they only prevent destructive interference. By its very nature, this exercise requires on-going government regulation of the technology because today's "white space" is tomorrow's operating region for the licensee. Further encumbering the licenses will

\footnotetext{
${ }^{15}$ See Lehr, Gillett and Merino (2003) for discussion of cognitive or software radios.

${ }^{16}$ See Farber and Faulhaber (2002).
} 
hamper the emergence of secondary markets and will retain regulatory constraints that the move to flexible licensing is intended to eliminate. Second, in part because of the valid concern just mentioned and in part because of a resistance to any reduction in existing rights, there is strong opposition to the creation of such secondary use access rights for unlicensed devices. It might be easier to mobilize support for an additional allocation of dedicated unlicensed spectrum than for underlay or overlay rights. Third, UWB and cognitive radio technologies are still under development and their technical and commercial viability remain speculative. While it seems likely that these technologies will be important, licensees have valid concerns that sharing licensed spectrum with these will cause interference and management problems that might be better addressed by segregating these access models.

In the next section, I explain why unlicensed access model is important and should be regarded as appropriate for more than (in addition to) secondary access.

\section{Benefits of Unlicensed Spectrum Model}

As noted earlier, the move to flexible licensing is more consistent with a smooth evolution of regulatory policy from a model of centralized command and control to management by markets. However, sole reliance on this model (or relegating unlicensed users to only secondary access) would be a mistake. In this section, I discuss three important reasons for why the unlicensed model is attractive and likely to be increasingly important in the future.

\section{A. More consistent with trajectory of technology and market trends}

While the past may belong to a world of exclusive-use licenses, the future belongs increasingly to unlicensed. The unlicensed model is more consistent with the trajectory of technology and with market trends.

First, the trajectory of technological innovation in wireless services has made frequencies more substitutable. It is much easier to accommodate divergent uses in the same frequency band or to support the same uses at different frequency bands. Innovations in signal processing, antenna design, and modulation schemes have significantly increased the information carrying capacity of existing spectrum. Collectively these innovations have made spectrum less scarce.

While scarcity remains an issue, it is reasonable to suppose that the acute (largely artificial) scarcity that has characterized spectrum in recent years and underlies the high prices paid for spectrum at auction will be greatly reduced by appropriate reform. Such reform should and is likely to include a substantial increase in the amount of commercial spectrum managed via flexible licenses that can be traded on secondary markets. In this future world, maximizing spectral efficiency may no longer be as important as other considerations in the choice of management regime. For example, it may be more important to provide low-cost entry for new services or technologies. Or, to minimize spectrum transaction costs. As spectrum scarcity declines, the opportunity cost of 
spectrum declines and the relative importance of any transaction costs becomes more important. Ceteris paribus, the reduction in scarcity makes unlicensed more attractive.

Second, technology has been evolving so as to decouple wireless services from specific frequency bands. Intelligence is moving into all components of radio systems. Smart radio systems enable more robust, lower power, higher data rate communications over longer distances. Smart receivers increase options for managing interference, empowering more decentralized and distributed models for spectrum management. Software radios able to support multiple protocols and capable of dynamically shifting frequencies reduce the extent to which services and much of network infrastructure (e.g., towers and wired connections to base stations) are co-specialized with particular frequencies.

Third, the transition to wireless broadband will increase the dynamic range of bandwidth requirements that will need to be supported. This will increase the opportunity cost associated with retaining the excess bandwidth needed to support high peak-toaverage data rate traffic, increasing the benefits from sharing access to spectrum across multiple service providers.

Fourth, and finally, providing for more unlicensed is consistent with the growth of a more complex and heterogeneous wireless landscape. The future will not be comprised of a single wireless network or technology. Rather there are likely to be multiple types of networks operating at a variety of geographic ranges, offering a range of capabilities, and implemented using many protocols and architectures. In this multi-protocol chaos, systems will adapt to become more tolerant of this sort of world. The transition to smart radio systems is more consistent with unlicensed and with the emergence of this heterogeneous wireless future.

\section{B. Helps promote competition and innovation}

Allocating additional spectrum for unlicensed use also helps promote competition by making it easier for new services, networks, and architectures to deploy. Unlicensed facilitates the distributed and decentralized growth of equipment or edge-based networking technologies such as ad hoc or mesh networks. Islands of connectivity can be created by end-users and then linked together to virally grow alternative infrastructure. This could be an important source for last-mile competition for access services.

Exclusive licenses create the potential for artificial scarcity which might be used to foreclose new technologies or competition that threatens incumbent providers. As long as spectrum is scarce (and the presumption of such scarcity is used to justify the need for exclusive licensing in the first place), such ex ante foreclosure remains a risk. Additionally, there is a risk of ex post foreclosure. Technologies or services which turn out to be more successful than originally anticipated are liable to expropriation by a spectrum licensee if it turns out that the technology/service turns out not to be frequency agile. Control of the spectrum might be used to expropriate the value of customer premise equipment. Consider whether WiFi would have been as successful if purchasers of equipment faced uncertain future charges from third party spectrum licensees to operate 
WLAN base stations. While it is both possible and likely that equipment makers can purchase spectrum also, unlicensed will remain an important option to foster small scale and experimental entry.

Unlicensed will also foster adoption of interference-tolerant, intelligent radio systems. To the extent operation in unlicensed spectrum suffers from congestion, the systems that will thrive there will be ones that are more interference tolerant. This may include both uses that simply care less about interference protection and so do not wish to pay for the added benefits afforded by operation under in exclusive licensed spectrum, or embody technologies that make it feasible to operate in uncertain and fluctuating congestion environments. Technologies that survive when there is a risk of a "Tragedy of the Commons" are those that either do not care or can ameliorate its impacts.

\section{Helps future-proof policy}

Providing for unlicensed access both via more dedicated unlicensed and through targeted easements to allow unlicensed secondary access via underlays and overlays helps future-proof policy.

The future of wireless services and technology is too uncertain to determine what services should exist in which frequency bands. The structure of the communications industry is too uncertain to determine which business models or market structures will best support future networking needs.

If the debate over licensed versus unlicensed spectrum does nothing else, it demonstrates that there is consensus that the two models imply different regulatory and industry economics (even if disagreements continue as to which is better). The availability of unlicensed spectrum provides a safety-valve for competitive entry and business models that may be less-successful in flexible licensed spectrum. Some of these may choose to migrate to licensed spectrum when they grow up. Because spectrum at different frequencies has very different physical properties (e.g., antenna size, propagation properties, line of sight requirements), it is important to adequately provide for both unlicensed and licensed spectrum models in the higher-value, lower-frequency spectrum to ensure that the choice of regulatory model does not bias the evolution of the wireless future.

\section{Conclusions}

While there is growing consensus in many countries that there is a need to transition from command \& control models for spectrum management to more marketbased modes, implementing reform is providing very difficult. Holders of exclusive licenses do not want to see their rights circumscribed by the granting of new secondary use easements. They do not want to be forced to pay more for spectrum they already occupy or to face competition from competitors able to exploit any new allocation of lower cost commercial spectrum. Entrants and consumer advocates are concerned that the incumbents may benefit unfairly if flexibility is provided for their exclusive licenses without compensation. All parties are bargaining over the allocation of surplus, the 
windfall gains, that are anticipated by the transition to a more efficient spectrum management regime. Not surprisingly, managing the transition to a new regime is difficult. Allocating additional spectrum to both flexible licensed and unlicensed uses can make this easier by helping to coopt the opposition.

The future of wireless services will be for spectrum to be shared more intensively than it is today in most bands (with the exception of mobile service bands which in many markets are already at capacity). However, even with mobile services, it is reasonable to expect that it will be advantageous to share access to 3G spectrum, rather than allocate all of the spectrum needed for each potential provider to support the full spectrum of anticipated services. These carriers may wish to manage their collective $3 \mathrm{G}$ spectrum as a private commons (i.e., shared access among a closed user community). Whether spectrum is shared via private or public commons, unlicensed has a role to play in helping to develop the necessary technologies and business models.

If we fail to allocate additional spectrum for unlicensed use in the highest value, lower frequency spectrum, we will never know what might have been. If we are successful in expanding commercial access to flexible use licensed spectrum but not of unlicensed, we will simply never observe what sorts of technologies or business models might have developed. We will have locked in only those equilibria that are possible in a world of exclusively licensed spectrum. No one will be able to measure the value of technologies that never develop or come to market. Because this world is closer to the status quo we have today, and is premised on a high marginal cost for incremental spectrum, if this model continues to result in significant artificial scarcity, it will appear as if the arguments in favor of licensed spectrum were correct.

The success of WiFi in recent years should caution policymakers against foreclosing alternative business models. WiFi operates in the $2.4 \mathrm{Ghz}$ band that was considered garbage spectrum because it was already so encumbered with noise (e.g., microwave ovens). The global growth of WiFi equipment markets, networks, and services has been exponential; and while it still accounts for substantially less activity than is associated with mobile wireless services and may not be suitable for building basic access infrastructure (i.e., not "carrier-grade"), it has had a profound impact on the trajectory of all wireless services. It has caused industry participants all along the value chain to rethink wireless futures. Mobile carriers are now looking at ways to incorporate WiFi access into their $3 \mathrm{G}$ architectures and new types of service providers (e.g., WISPs and power companies) are using $\mathrm{WiFi}$ as part of their last-mile alternative infrastructure plans. ${ }^{17}$ To ensure that this sort of innovation continues, and to realize the benefits of significant spectrum management reform it is important that the role for unlicensed access models be expanded.

References:

${ }^{17}$ See Lehr, Sirbu and Gillett (2004) and Lehr and McKnight (2003). 
Benkler, Yochai (2002), "Some Economics of Wireless Communications," 16 Harvard Journal of Law and Technology 25 (2002).

Cave, Martin (2002), “Review of Radio Spectrum Management,” Independent Review Report prepared for Department of Trade and Industry, United Kingdom, March 2002 (available from: http://www.ofcom.org.uk/static/archive/ra/spectrum-review/index.htm).

Comments of 37 Concerned Economists (2001), In the Matter of Promoting Efficient use of Spectrum through Elimination of Barriers to the Development of Secondary Markets, Before the Federal Communications Commission, WT Docket No. 00-230, February 7, 2001.

Faulhaber, Gerald R., and David Farber (2002), "Spectrum Management: Property Rights, Markets, and the Commons,” AEI-Brookings Joint Center, Working Paper 02-12 (December 2002).

Hazlett, Thomas (2001) “The Wireless Craze, The Unlimited Bandwidth Myth, The Spectrum Auction Faux Pas, and the Punchline to Ronald Coase's "Big Joke": An Essay on Airwave Allocation Policy" Harvard Journal of Law and Technology (Spring 2001).

Ikeda, Nobuo and Lixin Ye (2003), "Spectrum Buyouts: A Mechanism to Open Spectrum," Draft paper, October 17, 2003.

Kolodzy, Paul (2002), Spectrum Policy Task Force, Office of Engineering \& Technology, Federal Communications Commission, November 2002.

Kwerel, Evan and John Williams (2002), "A Proposal for a Rapid Transition to Market Allocation of Spectrum," OPP Working Paper \#38, Federal Communications Commission, November 2002.

Lehr, William (2004), "Economic Case for Dedicated Unlicensed Spectrum Below 3GHz,” paper prepared for New America Foundation Conference, April 2004.

Lehr, William and Lee McKnight (2003), "Wireless Internet Access: 3G vs. WiFi?," Telecommunications Policy, 27 (2003) 351-370.

Lehr, William, Sharon Gillett, and Fuencisla Merino (2003), "Software Radio: Implications for Wireless Services, Industry Structure, and Public Policy," Communications and Strategies, IDATE, Issue 49 ( $\left(1^{\text {st }}\right.$ Quarter 2003) $15-42$.

Lehr, William, Marvin Sirbu, and Sharon Gillett (2004), "Municipal Wireless Broadband: Policy and Business Implications of Emerging Access Technologies," paper prepared for "Competition in Networking: Wireless and Wireline," London Business School, April 1314, 2004.

Lessig, Lawrence (2001), The Future of Ideas: The Fate of the Commons in a Connected World, New York: Random House, 2001. 
Noam, Eli (1995), “Taking the Next Step Towards Open Spectrum Access,” IEEE Communications Magazine, 33 (1995) 66.

Ofcom (2004), "Spectrum Framework Review: A consultation on Ofcom's views as to how radio spectrum should be managed," UK Communications Regulatory Authority November 23, 2004 (available at: http://www.ofcom.org.uk/consultations/current/sfr/sfr.pdf?a=87101) .

Reed, David (2002), "How Wireless Networks Scale: The Illusion of Spectrum Scarcity," presentation slides to FCC Technology Advisory Council, Washington, DC, April 26, 2002 (available at:

http://www.jacksons.net/tac/Spectrum\%20capacity\%20myth\%20FCC\%20TAC.pdf)

Werbach, Kevin (2003), "Radio Revolution: The Coming Age of Unlicensed Wireless," white paper prepared for New America Foundation, Washington, DC, December 2003. 